\title{
Measurement of Cross-Section Geometry Effects on Electron Collection to Long Probes in Mesosonic Flowing Plasmas
}

\author{
Éric Choinière* and Brian E. Gilchrist ${ }^{\dagger}$ \\ University of Michigan, Ann Arbor, Michigan 48109 \\ Sven G. Bilén ${ }^{\ddagger}$ \\ The Pennsylvania State University, University Park, Pennsylvania 16802 \\ Keith R. Fuhrhop ${ }^{\S}$ \\ University of Michigan, Ann Arbor, Michigan 48109
}

\begin{abstract}
This paper presents the analysis and comparison of measured electron current collection to cylindrical, solid tape, and slotted tape electrodynamic-tether samples in a mesosonic flowing plasma. A Hall thruster was used to simulate a flowing unmagnetized space plasma in a large $6 \mathbf{m} \times 9 \mathbf{m}$ vacuum chamber. Guarded tether samples were designed to mitigate end effects. Plasma parameters were determined based on the ion saturation and electron retardation regimes of a Langmuir probe's current characteristics. Solid tape samples with effective widths spanning from 4.9 to 41.9 Debye lengths, and slotted tapes with line spacings spanning from 1.4 to 13.2 Debye lengths were tested. Several conclusions can be drawn from the analysis of the results: 1) The plasma flow leads to current enhancements over that predicted by the orbital-motion-limited theory; 2) the electron collection efficiency of solid tapes (on a per area basis) decreases as the width of the tape is increased; 3) beyond a threshold bias close to the beam energy, solid and slotted tapes both collect more current when oriented transverse to the flow; 4) equivalent width slotted tapes are more efficient electron collectors than solid tapes on a per area basis; 5) our data suggests the electron collection efficiency of slotted tapes decreases with increasing line spacing until a possible minimum efficiency is attained, beyond which it is expected to start increasing again. The minimum was attained in the case of the samples oriented transverse to the flow, but not in the case of the samples aligned with the flow, for which the critical spacing is likely higher due to an increased sheath interaction radius of each line caused by the elongation of the sheath associated with plasma flow.
\end{abstract}

\section{Background}

$\mathbf{T}$ HE use of a bare section of a space electrodynamic tether as an electron-collection device has been shown ${ }^{1}$ to be a promising alternative to endbody electron collectors, provided that electrons are collected in a quasi orbital-motion-limited regime.

The bare tether concept will be first tested during NASA's Propulsive Small Expendable Deployer System (ProSEDS) mission, ${ }^{2}$ to be flown in the near

\footnotetext{
* Graduate Student, Radiation Laboratory, EECS Department, AIAA Student Member, echoinie@umich.edu

${ }^{\dagger}$ Professor, Electrical Engineering and Space Sciences, AIAA Senior Member, gilchrst@umich.edu

${ }^{\ddagger}$ Assistant Professor, Electrical Engineering Department, AIAA Member, sbilen@psu.edu

$\S$ Graduate Student, Radiation Laboratory, EECS Department, AIAA Student Member, kfuhrhop@umich.edu
}

future, and is being considered for future missions. Current bare tether designs, such as the one used in the ProSEDS mission, use a small, closely packed cross-section of wires or even a single wire as the anode. In future designs, addressing concerns such as survivability to collisions with micro-meteoroids and space debris will require the use of distributed or sparse tether cross-section geometries, which could span tens of Debye lengths, depending on plasma density. ${ }^{3}$

Since the merits of bare tethers are closely related to the efficiency of the orbital-motion-limited regime, one needs to consider how these new distributed or sparse geometries will perform in terms of current collection, as compared to thin cylinders. In addition, the effect of the high-speed flow on the electron collection to these alternative geometries as well as to thin cylinders has yet to be clearly understood. Ultimately, designers will need to know how to configure a tether for adequate survivability and 

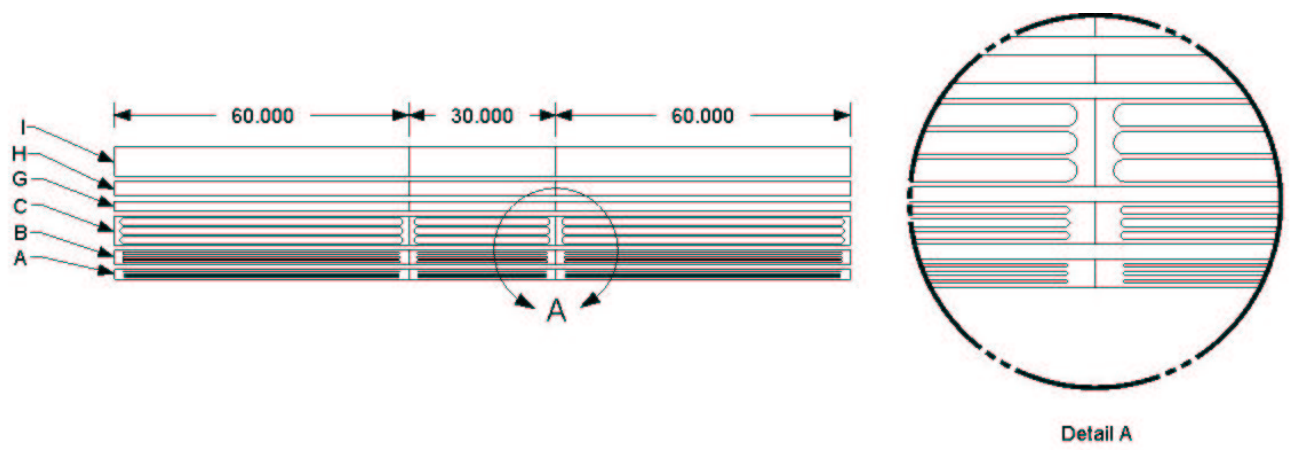

\begin{tabular}{|c|l|c|l|}
\hline Sample & Description & $\begin{array}{c}\text { Width } \\
(\mathrm{mm})\end{array}$ & Feature Description \\
\hline \hline A & 4 lines, 3 slots, 28\% porosity & 1.95 & Slot gap: $0.20 \mathrm{~mm}$, line width: $0.34 \mathrm{~mm}$ \\
\hline B & 4 lines, 3 slots, 50\% porosity & 2.89 & Slot gap: $0.51 \mathrm{~mm}$, line width: $0.34 \mathrm{~mm}$ \\
\hline C & 4 lines, 3 slots, 75\% porosity & 5.95 & Slot gap: $1.53 \mathrm{~mm}$, line width: $0.34 \mathrm{~mm}$ \\
\hline G & Narrow Solid Tape & 1.95 & N/A \\
\hline H & Medium Solid Tape & 2.89 & N/A \\
\hline I & Wide Solid Tape & 5.95 & N/A \\
\hline
\end{tabular}

Fig. 1 Drawing and description of the six guarded tether samples shown before assembly. The lengths indicated in the drawing are in $\mathrm{mm}(30-\mathrm{mm}$ probe, $60-\mathrm{mm}$ guards)

minimum mass for example.

In this paper, the orbital motion limit will be used as a baseline when comparing the current collection results for different sample geometries and sizes. The OML electron current collected by a thin cylinder is given by ${ }^{4}$

$$
\begin{aligned}
I=A_{p} & \underbrace{n_{e} e \sqrt{\frac{e T_{e}}{2 \pi m_{e}}}}_{I_{\text {the }}}\left\{\frac{2}{\sqrt{\pi}} \sqrt{\frac{V-V_{p}}{T_{e}}}\right. \\
& \left.+\exp \left(\frac{V-V_{p}}{T_{e}}\right) \operatorname{erfc}\left(\sqrt{\frac{V-V_{p}}{T_{e}}}\right)\right\},
\end{aligned}
$$

with $\operatorname{erfc}(x)=\frac{2}{\sqrt{\pi}} \int_{x}^{\infty} e^{-t^{2}} d t$. Eq. (1) is approximated, for $V-V_{p}>2 T_{e}$, by:

$$
I=A_{p} \underbrace{n_{e} e \sqrt{\frac{e T_{e}}{2 \pi m_{e}}}}_{I_{\text {the }}} \frac{2}{\sqrt{\pi}} \sqrt{1+\frac{V-V_{p}}{T_{e}}}
$$

where $V$ is the applied voltage, $V_{p}$ is the plasma potential, $T_{e}$ is the electron temperature in $\mathrm{eV}, A_{p}$ is the cylinder area, and $I_{\text {the }}$ is the electron thermal current, given as a function of the electron number density $n_{e}$ (in $\left.m^{-3}\right)$, electron mass $m_{e}$, and electron charge $e$. In an effort to ease comparisons with OML theory, our results are presented in a normalized format, showing the normalized current $I_{n}=I /\left(A_{p} I_{\text {the }}\right)$ as a function of the normalized voltage $\hat{V}=\left(V-V_{p}\right) / T_{e}$, which in the case of OML yields the simple equation:

$I_{n}=\frac{2}{\sqrt{\pi}} \sqrt{\hat{V}}+\exp (\hat{V}) \operatorname{erfc}(\sqrt{\hat{V}}) \approx \frac{2}{\sqrt{\pi}} \sqrt{1+\hat{V}}$.

For large voltages, this equation is independent of the temperature $T_{e}$, since both the right-hand side and left-hand side are then proportional to $1 / \sqrt{T_{e}}$. This normalization will allow us to directly compare the OML theory, which only applies to thin cylinders in non-flowing plasmas, with our experimental results involving a flowing plasma and various tether geometries.

In a previous investigation, ${ }^{5}$ it was determined that tape tethers with widths up to 10 Debye lengths would perform close to an equal-area reference cylinder, and that the perpendicular tape orientation would consistently outperform the parallel orientation in terms of collected current.

In the present communication, we describe the results of a new set of chamber tests that addresses questions of end effects and plasma source current limitations discussed in Ref. 5 , in addition to testing a larger breadth of "solid tape" widths and a new "slotted tape" geometry with various porosities. The issue of end effects was addressed by adding guards to the tether samples, which are described below. The question of possible plasma source current limitations, that was raised in our previous publication, ${ }^{5}$ was resolved by modifying the plasma source parameters, as discussed in a later section. 


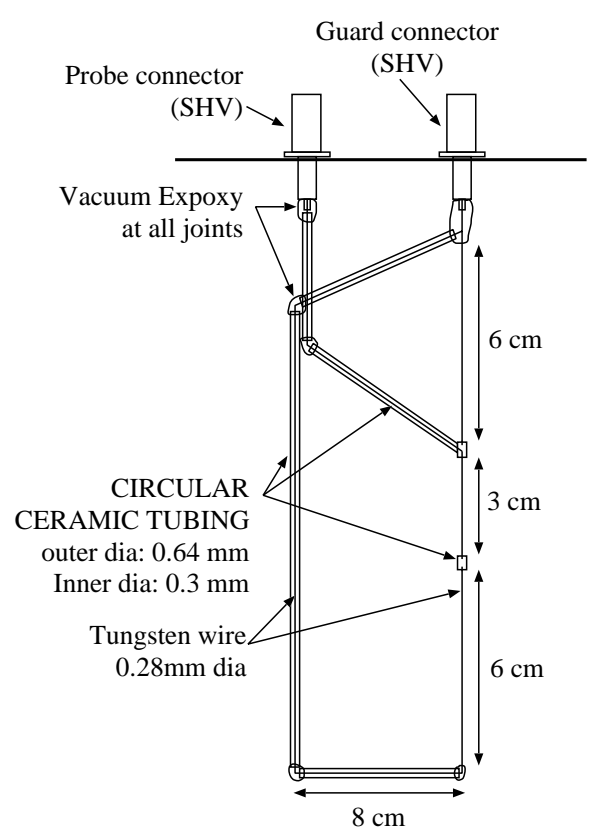

(a)

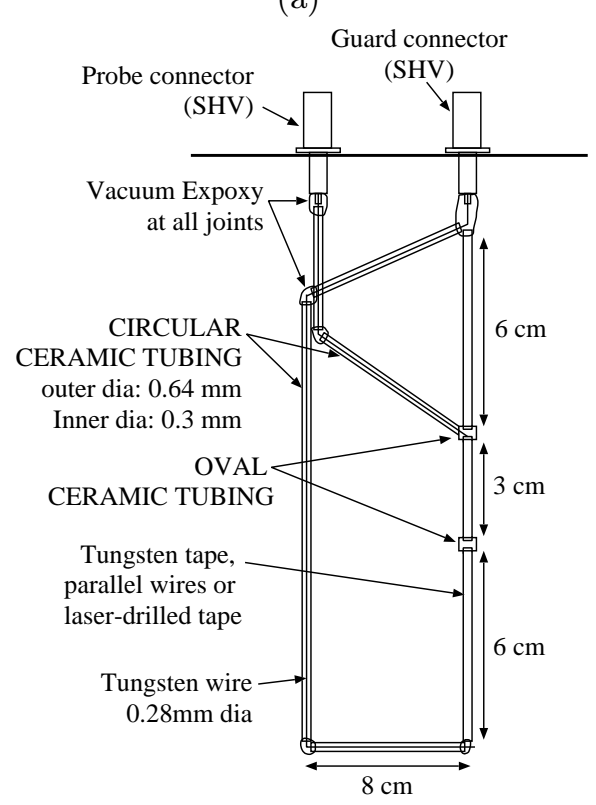

(b)

Fig. 2 Assemblies of the reference cylinder(a) and tape (b, solid \& slotted) guarded tether samples.

\section{Design and Assembly of Solid and Slotted Tape Tether Guarded Samples}

The tether samples tested here, in addition to a thin cylindrical reference sample, included solid tape samples in three different widths, and slotted samples of equivalent widths and corresponding to three different porosity levels. The details of these designs, as well as a drawing of the samples and their char-

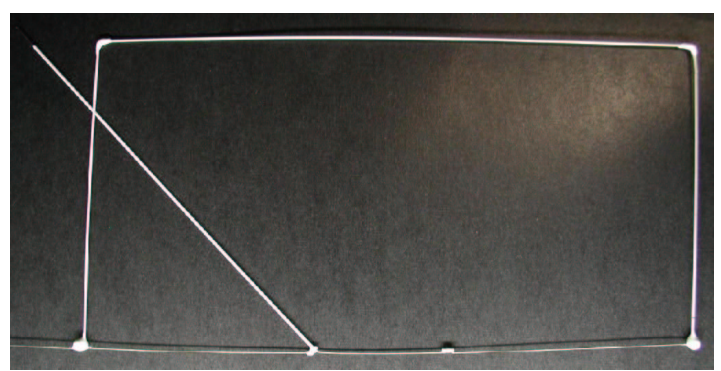

(a)

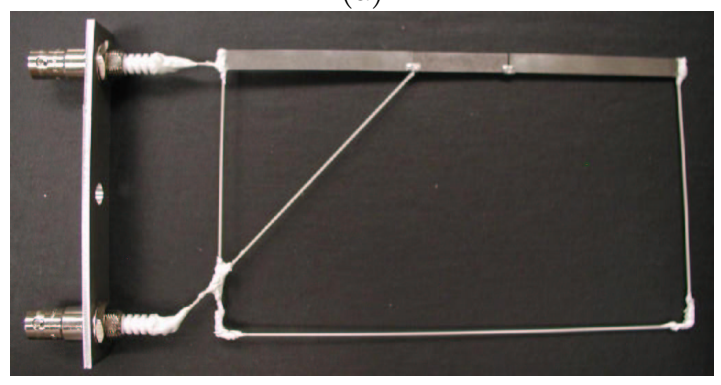

(b)

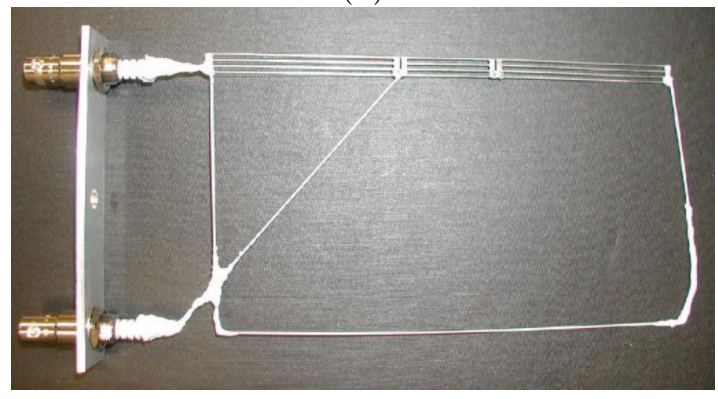

(c)

Fig. 3 Pictures of three typical tether samples: (a) the $0.28 \mathrm{~mm}$-diameter reference cylinder, (b) the 5.95-mm wide solid tape, and (c)the 75\%porous wide slotted tape.

acteristic sizes, are shown in Fig. 1. Each of these 7 samples ( 1 ref. cyl. +3 solid tapes +3 slotted tapes) was tested in two different orientations, parallel and perpendicular to the plasma flow, at three different distances from our plasma source. Tungsten metal was used for all samples to ensure that they would endure the expected high temperatures that are caused by the collection of high-energy electrons to the samples' surfaces. Nonetheless, a low duty cycle pulsing of the applied voltages was necessary to allow the samples to cool off thus preventing melting; details of this procedure are reported in Ref. 5 .

The effective diameter of the reference cylinder and effective widths of the three tape samples, for the plasma densities tested here, are given in Table 1 in terms of the local mean-free-path for the three chamber positions that were tested. The reference cylinder's diameter, spanning from 0.7 to 2.0 De- 


\begin{tabular}{|c||c|c|c|c|}
\hline & Ref. & \multicolumn{3}{|c|}{ Solid Tape } \\
\cline { 3 - 5 } Pos. & Cyl. & Narrow & Medium & Wide \\
\hline $75 \mathrm{~cm}$ & 2.0 & 13.8 & 20.4 & 42.0 \\
\hline $160 \mathrm{~cm}$ & 1.1 & 7.4 & 11.0 & 22.6 \\
\hline $300 \mathrm{~cm}$ & 0.7 & 4.9 & 7.2 & 14.9 \\
\hline
\end{tabular}

Table 1 Effective diameter of the reference cylinder and widths of the three solid tapes at all three locations, in terms of the local Debye length.

\begin{tabular}{|c||c|c|c|}
\hline \multirow{2}{*}{\multicolumn{1}{|c||}{ Position }} & \multicolumn{3}{c|}{ Slotted Tapes by Porosity } \\
\cline { 2 - 4 } & $\mathbf{2 8 \%}$ & $\mathbf{5 0 \%}$ & $\mathbf{7 5 \%}$ \\
\hline \hline $75 \mathrm{~cm}$ & 3.8 & 6.0 & 13.2 \\
\hline $160 \mathrm{~cm}$ & 2.1 & 3.2 & 7.1 \\
\hline $300 \mathrm{~cm}$ & 1.4 & 2.1 & 4.7 \\
\hline
\end{tabular}

Table 2 Effective center-to-center spacing between neighboring lines as a function of sample porosity at all three locations, in terms of the local Debye length

bye lengths depending on position, is sufficiently thin to collect electron current under conditions close to that of the OML regime in a non-flowing plasma. The effective solid tape widths spanned from 4.9 to 42 Debye lengths, which extends our previous range of tested widths. ${ }^{5}$

As for the three slotted samples, they were designed with the same overall widths as their solid counterparts; this strategy allowed us to compare solid and porous samples spanning equivalent widths. In addition, the design is such that each of the four lines on every slotted sample has the same perimeter as our reference cylinder, allowing one to consider the effects and measure the extent of sheath interactions. The effective center-to-center spacings of the slotted samples are given in Table 2.

Since our interest is very long electrodynamic tethers, a technique was sought to mitigate any probe end effects. To this effect, guards were included in all of our tether sample assemblies. Each guard is essentially identical to the center section and is biased to the same potential. The guards, which are $6 \mathrm{~cm}$ in length while the center probe itself is $3 \mathrm{~cm}$ long, are shown in Fig. 1 together with the probes. The effect of the guards is to extend the cylindrical sheath to the full length of the sample, that is, five times the length of the center probe on which current is measured.

Schematics of the full assemblies of the guarded tether samples are shown in Fig. 2. Due to the very small thickness of the tungsten samples under consideration here (0.1-mm thick), it was not physically possible to feed the center probe using a feeding wire that would have been inserted in one of the guards,

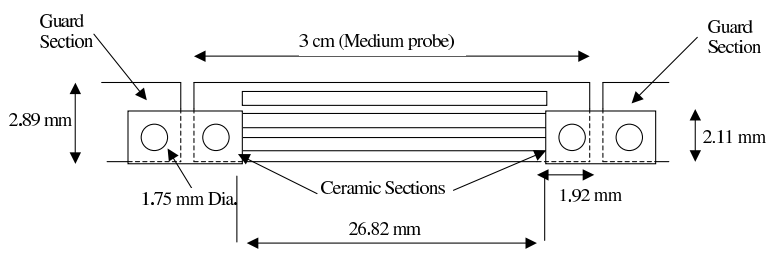

Fig. 4 Example of the ceramic attachment used on all solid and slotted tape samples to attach the probe and guards while preserving electrical isolation.

as is typically done on some larger tri-axial Langmuir probes. Instead, the center feed wire runs through an oblique ceramic tube and connects to the center probe on one of its ends. On all samples, the feed wires to both the guards and the probe were both soldered to the center conductor of a bulk-head safe high voltage (SHV) connector; the connectorsample interfaces were then covered with vacuum epoxy. The aluminum support structure provided a localized ground.

The probe and guards also had to be physically attached but electrically insulated from each other. Ceramic joints were used to this effect, an example of which is shown in Fig. 4, and were attached to the tungsten probes and guards using stainless-steel machine screws that were then carefully sanded down into a flat surface, in order to best emulate the surface of the sample. The portion of the surface area of the ceramic joint covering the tungsten probe and not covered by the screw head was accounted for in the calculation of the total area of each probe.

Fig. 3 shows pictures of three of our tether sample assemblies: the reference cylinder, the wide solid tape, and the $75 \%$-porous wide slotted tape. The solid and slotted samples are shown with both SHV connectors installed, while the reference cylinder sample is shown prior to the installation of the connectors.

\section{Vacuum Chamber Setup and Plasma Source Characteristics}

Our vacuum chamber tests were performed using the Large Vacuum Test Facility (LVTF), a 9 meter by 6 meter cylindrical stainless-steel-clad tank located within the Plasmadynamics and Electric Propulsion Laboratory at the University of Michigan. For this experiment, four of the seven available nude cryopumps were used to reach a high vacuum.

Fig. 5 shows a diagram of the experimental setup within the LVTF. Two positioning tables were used to change the separation distance between the thruster and sample plane and to locate the sample under test directly along the thruster's centerline. 


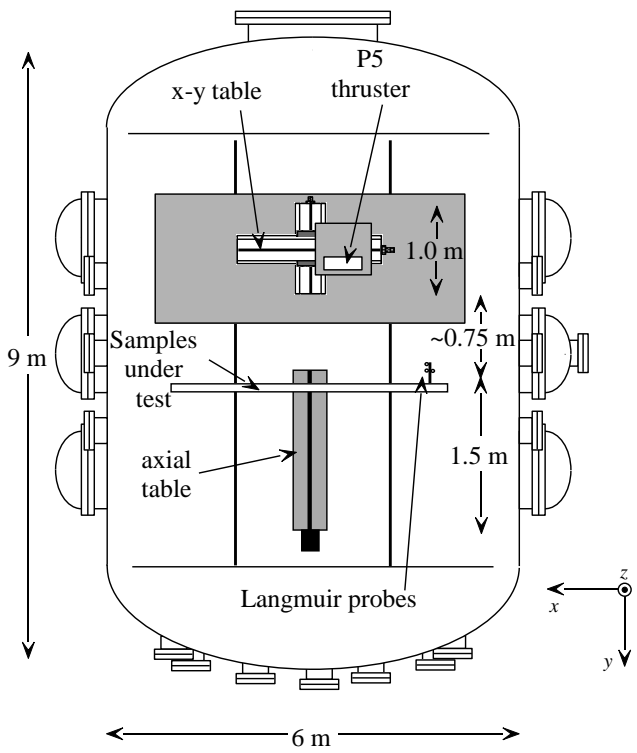

Fig. 5 Experimental setup in the Large Vacuum Test Facility (LVTF) at the Plasmadynamics and Electric Propulsion Laboratory (PEPL).

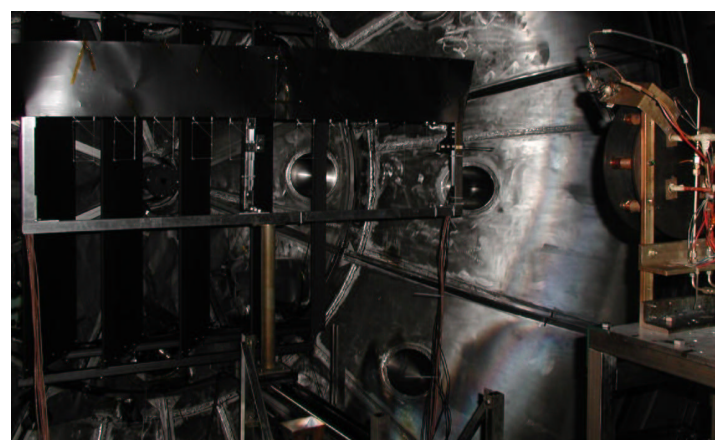

Fig. 6 Picture of the guarded sample support structure and the P5 hall thruster used as a highspeed plasma source

The thruster was mounted on an $x-y$ table that could move axially over a 1.0-m range and over a sufficient radial range to cover all samples. The samples were mounted on an aluminum frame that was connected to an axial table that could span a $1.5-\mathrm{m}$ axial range. Combined table movement allowed thruster-sample separation distance to change from $0.75 \mathrm{~m}$ to $3 \mathrm{~m}$; our tests were performed at $0.75 \mathrm{~m}, 1.60 \mathrm{~m}$ and 3.00 $\mathrm{m}$ from the thruster. Changing separation distance was the primary mechanism for changing the plasma density seen at the sample plane.

Fig. 6 shows an overall picture of the aluminum structure supporting our tether samples and Langmuir probes, together with the Hall thruster used as a plasma source. The latter is a $5 \mathrm{~kW}$-class Hall thruster named "P5", which was developed by the Plasmadynamics and Electric Propulsion Labora-

\begin{tabular}{lc} 
Maximum Chamber Pressure & $18.8 \mu$ torr \\
Discharge Voltage, $V_{d}$ & $100 \mathrm{~V}$ \\
Discharge Current, $I_{d}$ & $12.5 \mathrm{~A}$ \\
Inner Magnet Current, $I_{i m}$ & $3.0 \mathrm{~A}$ \\
Outer Magnet Current, $I_{o m}$ & $2.0 \mathrm{~A}$ \\
Cathode Voltage, $V_{c}$ & {$[-17,-18] \mathrm{V}$} \\
Heather Voltage, $V_{\mathrm{htr}}$ & $8.3 \mathrm{~V}$ \\
Anode Flowrate, $\dot{m}_{a}$ & $112.1 \mathrm{sccm}$ \\
Cathode Flowrate, $\dot{m}_{c}$ & $6.0 \mathrm{sccm}$ \\
\hline
\end{tabular}

Table 3 Operating Parameters of the Plasma Source (P5 Hall Thruster)

tory and the Air Force Research Laboratory; more detail is given by Haas et al. ${ }^{6}$ For these tests, the thruster was set at off-nominal conditions in order to lower the plasma velocity and density seen along the thruster's axial direction. Its operating conditions are given in Table 3. The primary changes in those settings from the ones used previously ${ }^{5}$ are the discharge current, which was raised to $12.5 \mathrm{~A}$, up from $5.3 \mathrm{~A}$ and $4 \mathrm{~A}$ and, consequently, the anode flowrate, which had to be raised to $112.1 \mathrm{sccm}$ to support the increased discharge current.

Assessment of the emitted beam energy was estimated using two different techniques. Laser-induced fluorescence (LIF) measurements have provided an estimate of $42.9 \mathrm{eV},{ }^{7}$ whereas Langmuir probe (LP) measurements in the ion saturation regime (discussed in the next section) have yielded a value of $25.0 \mathrm{eV}$. More detail regarding the LIF measurements is given in Ref. 5 and Ref. 8. It should be noted that the LP value of $25.0 \mathrm{eV}$ is close to, if not within, the bounds of the error on the LIFdetermined beam energy value, which is about $40 \%$, given that the reported typical error on the velocity determined using the multiplex technique is $20 \% .^{9}$

It should be noted that the values given for both the LIF and LP measurements were measured 75 $\mathrm{cm}$ away from the thruster on its centerline axis. According to the LIF measurement, the ions have an offset Maxwellian distribution, with a directed energy as given above, and a temperature of about $0.4 \mathrm{eV}$ at $75 \mathrm{~cm}$. The electron temperature, as determined by the LP measurements, varied as a function of position between $1.4 \mathrm{eV}$ and $1.8 \mathrm{eV}$ (see Table 4).

A schematic of the current-voltage measurement system is given in Fig. 7. We connected a Universal Voltronics BRC $20000 \mathrm{HV}$ power supply to the tether samples through a high-voltage relay box inside the chamber. The HV power supply was controlled via RS-232 by the computer controller running a custom virtual instrument (VI) under LabVIEW $^{\mathrm{TM}}$. The computer commanded the HV 


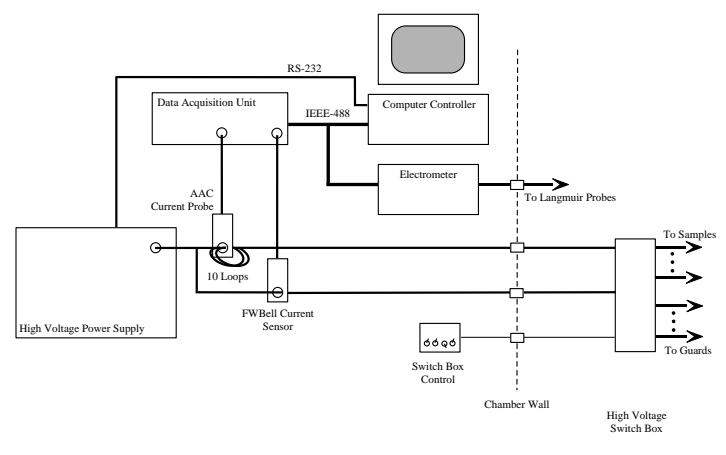

Fig. 7 Schematic of the computer-controlled high-voltage test equipment setup

power supply to a specified voltage and then quickly back to zero (within 50 to $100 \mathrm{~ms}$ ), followed by several seconds of cool-down, to minimize sample heating. Current measurement on the sample probes was achieved using an American Aerospace Controls 835-2-10 current sensor; increased current sensitivity was obtained by looping the HV supply line ten times through the sensor. The current to the sample guards was measured separately using a F.W.Bell ma-2000 current sensor. An HP 34970 data acquisition unit was used to measure the voltage signals generated by both current sensors. The data were recorded as triplets containing the applied voltage, the probe current, and the guard current.

\section{Plasma Parameter Measurements Using Negatively-Biased Langmuir Probes}

Plasma density, temperature, flow speed, and the fraction of beam ions to background ions were determined using a 4-cm long, vertically oriented (i.e., perpendicular to the flow) LP with a diameter of $0.28 \mathrm{~mm}$, as shown in Fig. 8 (same diameter as for our reference cylinder sample). All LP sweeps were performed using a Keithley 2410 source electrometer controlled via a custom LabVIEW ${ }^{\mathrm{TM}}$ script running on a personal computer.

Our plasma parameters, shown in Table 4, were extracted from the ion saturation(OML regime) and electron retardation regions of the IV characteristics using a LP oriented transverse to the direction of the flow. In the OML regime, there are several advantages to selecting the ion saturation as opposed to the electron saturation for parameter extraction. A cylindrical probe oriented transverse to the flow in a high-speed plasma is known to be virtually free of end effects. ${ }^{10}$ In addition, a simple but fairly accurate collection model is available that accounts for the velocity of the flow in that regime. ${ }^{11}$ By contrast, there are currently no accurate models

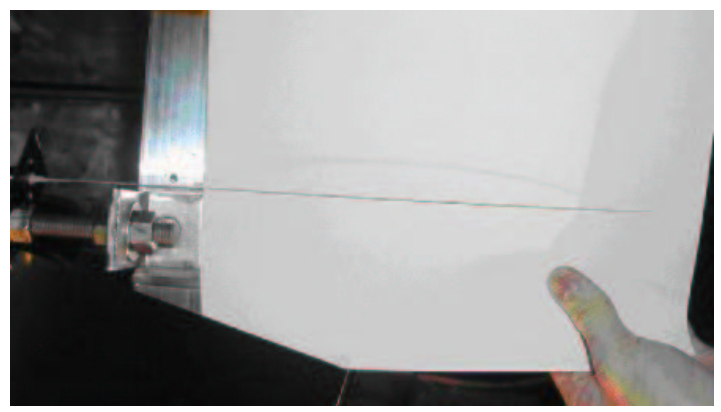

Fig. 8 Vertical Langmuir probe with ceramic sleeve, shown here horizontally due to space limitations. The bare part of the probe is $40 \mathrm{~mm}$ long and has a $0.28 \mathrm{~mm}$ diameter.

\begin{tabular}{|c||c|c|c|c|}
\hline Position & $\begin{array}{c}n_{e} \\
\left(\mathbf{m}^{-3}\right)\end{array}$ & $\begin{array}{c}T_{e} \\
(\mathbf{e V})\end{array}$ & $\begin{array}{c}\lambda_{D e} \\
(\mathbf{m m})\end{array}$ & $\mu_{b}$ \\
\hline \hline $75 \mathrm{~cm}$ & $4.95 \times 10^{15}$ & 1.80 & 0.14 & $95 \%$ \\
\hline $160 \mathrm{~cm}$ & $1.37 \times 10^{15}$ & 1.72 & 0.26 & $53 \%$ \\
\hline $300 \mathrm{~cm}$ & $0.51 \times 10^{15}$ & 1.47 & 0.40 & $32 \%$ \\
\hline
\end{tabular}

Table 4 Variation of the measured plasma parameters as a function of distance from the hall thruster. Measurements were performed using the ion saturation and electron retardation data from a transverse LP. The beam energy value determined using the $L P$ is $25 \mathrm{eV}$. The "beam fraction", $\mu_{b}$, indicates the fraction of all ions that are beam (high-speed) ions.

for the electron collection to an electron-attracting probe that can account for the plasma flow. In the mesosonic regime, where the plasma flow is much faster than the thermal ion velocity yet much smaller than the electron thermal velocity, important sheath asymmetries and elongations exist in the electronattracting mode that get stronger with the applied bias, which makes this a complex problem. One of the aims of the experimental work presented here is in fact to improve the understanding of the macroscopic effect of plasma flow on electron collection. An accurate model of electron collection in flowing plasmas is also currently being developed. ${ }^{12,13}$ Finally, using a negatively-biased LP provides an independent assessment of the plasma parameters, since all our test samples are biased positively.

The plasma parameter extraction is an iterative procedure that requires several iterations of the "ion saturation analysis" and "electron retardation analysis" described below, to reach convergence. At every step, the electron retardation analysis is performed on the electron current exclusively, by removing the ion saturation best fit from the current data. Conversely, the ion saturation analysis is performed on the ion current alone by removing the latest best fit to the electron retardation from the 


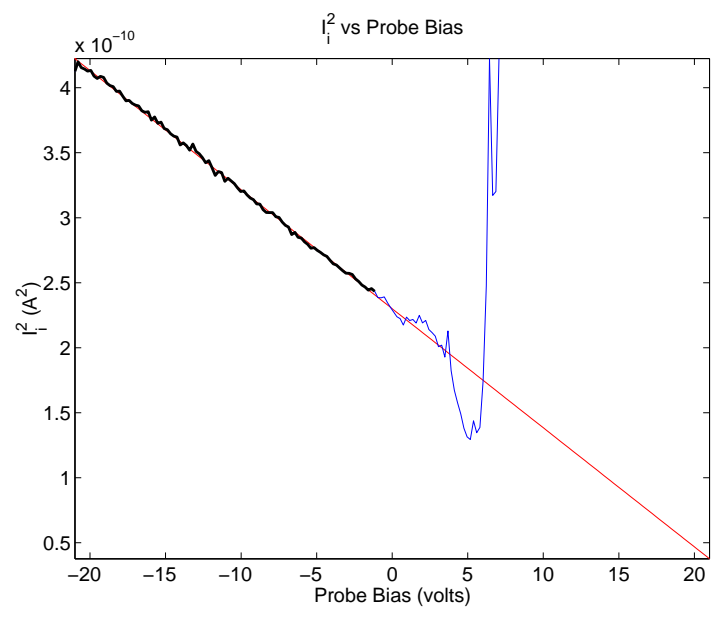

Fig. 9 Best fit of the $I_{i}^{2}$-vs- $V$ data in the ion saturation regime which is used to determine density, flow energy, and the fraction of beam ions.

measured current.

Ion Saturation Analysis: Density $n_{e}=n_{i}$, Flow Energy $F$, and High-Speed Fraction $\mu_{b}$

The OML ion current to a cylindrical probe biased negatively, $V-V_{p}<0$, in a plasma flowing at a velocity $U$ and a corresponding "flow energy" $F=$ $\frac{m_{i} U^{2}}{2 e}$ is given by: ${ }^{11}$

$$
I_{i}=\sqrt{2} \frac{A_{p} e^{1.5}}{\pi \sqrt{m_{i}}} n_{i} \sqrt{\underbrace{\alpha T_{i}}_{\approx 0}+F+V_{p}-V},
$$

where $\alpha$ is 1 for large biases, $V_{p}-V>>F$, and goes to $1 / 2$ for small biases. The slope of the best fit to the $I_{i}^{2}$-vs- $V$ LP data can be used to determine the plasma density $n_{e}=n_{i}$ (quasi-neutrality is strongly enforced within the plasma beam), while the offset allows one to determine an estimate for the quantity $\alpha T_{i}+F$. An example of this procedure is shown in Fig. 9. In our calculations of the flow energy $F$, we have neglected the first term $\alpha T_{i}$ since the ion temperature is very low, on the order of $0.4 \mathrm{eV}$, as determined from the laser-induced fluorescence (LIF) measurements described earlier.

An additional feature was added to the analysis in order to account for the presence of a background of low-energy ions that only have thermal energy (i.e., they are not flowing), in addition to the beam of directed ions. If we suppose that a fraction $\mu_{b}$ of the ions are beam ions and that the low-energy ions have a temperature $T_{\text {slow }}$, then the total ion current collected is given by:

$$
\begin{aligned}
I_{i}=\sqrt{2} \frac{A_{p} e^{1.5}}{\pi \sqrt{m_{i}}} n_{i}\left\{\mu_{b} \sqrt{\alpha T_{i}+F+V_{p}-V}\right. \\
\left.+\left(1-\mu_{b}\right) \sqrt{T_{\text {slow }}+V_{p}-V}\right\}
\end{aligned}
$$

Now, the slope of the resulting $I_{i}^{2}$ vs $V$ graph only varies slowly, as evidenced on Fig. 9, which clearly shows the linear behavior in the ion saturation region. We may still perform a best fit of the collected $I_{i}^{2}$ vs $V$, and determine the "apparent" ion density $n_{i, \text { app }}$ and flow energy $F_{\text {app }}<F$. Assuming the flow energy $F$ of the high-speed ion population is known, the actual ion density $n_{i}$ and the "high-speed fraction" $\mu_{b}$ both can be determined accurately, using the following set of formulas that were devised based on Eq. (5)

$$
\begin{aligned}
A & =\sqrt{T_{\text {slow }}+V_{p}-V_{\text {avg,fit }}} \\
B & =\sqrt{F+V_{p}-V_{\text {avg,fit }}} \\
C & =F_{\text {app }}+V_{p}-V_{\text {avg,fit }} \\
D & =\frac{1}{B}-\frac{1}{A} \\
\mu_{b} & =\frac{A-C / A}{C D+A-B} \\
n_{i} & =n_{i, \text { app } \frac{\sqrt{A B}}{\sqrt{\left(\mu_{b} B+\left(1-\mu_{b}\right) A\right)\left(\mu_{b} A+\left(1-\mu_{b}\right) B\right)}}}
\end{aligned}
$$

where $V_{\text {avg,fit }}$ is the average bias potential over which the best-fit was performed to obtain the values for $n_{i}$,app and $F_{\text {app }}$. There is a one-to-one map between the apparent flow energy $F_{\text {app }}$ and the actual high-speed ion fraction $\mu_{b}$ for given values of the high-speed energy $F$.

The value of the high-speed energy $F$ was calibrated such that the high-speed ion fraction $\mu_{b}$ is equal to 0.95 at the closest position to the thruster $(75 \mathrm{~cm})$, which is approximately in agreement with the energy distributions obtained using laser-induced fluorescence (LIF) at the same position. This procedure has yielded a beam energy of $F=25 \mathrm{eV}$, which is in within the bounds of the error of the LIF result $(42.9 \mathrm{eV})$, as discussed earlier. The high-speed fractions obtained for all three positions are shown in Table 4: $95 \%$ at $75 \mathrm{~cm}, 53 \%$ at $160 \mathrm{~cm}$, and $32 \%$ at $300 \mathrm{~cm}$. This fall-off of the fraction of beam ions can be best-fitted to an equivalent "beam ion survival characteristic distance" of $2.14 \mathrm{~m}$, which is just short of the theoretical charge-exchange mean free path of $2.5 \mathrm{~m}$ corresponding to the measured 


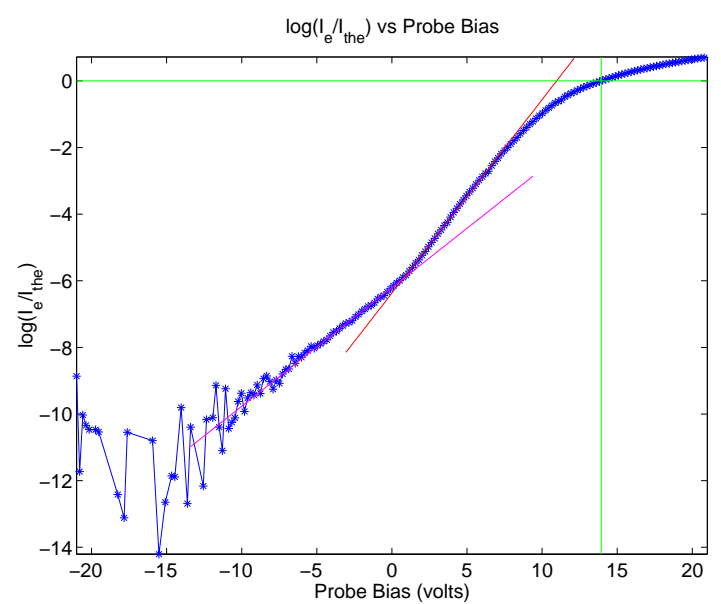

Fig. 10 Best fits in the electron retardation regime of a transverse-flow Langmuir probe. Two linear best-fits are performed on this semi-log plot. The one occurring at lower potential values corresponds to a population of warmer electrons, which account for just a few percent of the electron population.

background pressure, ion energy, and assuming a background neutral temperature of $350 \mathrm{~K}$. The falloff is actually due to a combination of effects, such as the beam loss through charge-exchange collisions and the geometrical divergence of the beam, which may explain the smaller value obtained.

Electron Retardation Analysis: Plasma Potential $V_{p}$ and Electron Temperature $T_{e}$

Variations in the effective workfunction on the surface of our LP (referred to as workfunction patchiness in Ref. 14) has turned out to be of major concern in our experimental data. It appears that a combination of possible surface contaminants and tungsten material inhomogeneities have resulted in a total effective workfunction variation of several $\mathrm{eV}$. Fig. 10 illustrates that effect, which leads to a departure from exponential behavior before the plasma potential is attained.

A simple numerical experiment on the theoretical current collection curves was performed in order to emulate the "blurring" effect of the workfunction variation. Fig. 11 shows the results of this numerical experiment in the "thin sheath" and "OML" limits. The dashed curves, shown in both linear and semilogarithmic formats, is the result of "blurring" the theoretical curves using a local averaging window of width equal to 4.8 times the electron temperature. The blurred thin sheath curve is shown to collect less than the thermal current $I_{\text {th }}$ at the plasma potential $\left(V / T_{e}=0\right.$ here $)$, while on the blurred OML curve, the collected current at plasma potential is still equal to
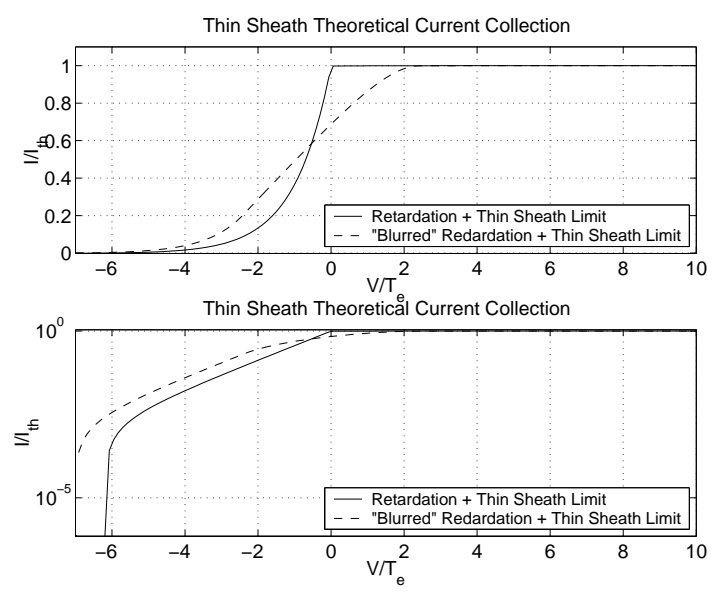

(a)
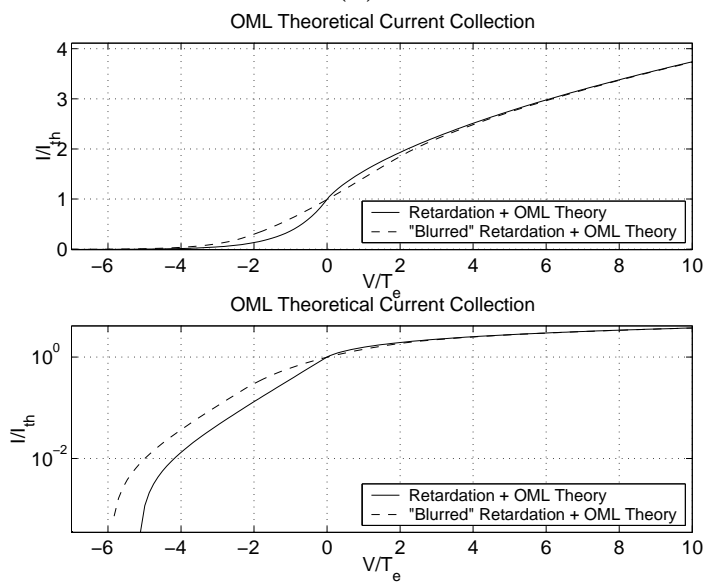

(b)

Fig. 11 Theoretical "blurring" of the theoretical current collection to probes in the (a)thin sheath and (b) $O M L$ limits

the thermal current $I_{\mathrm{th}}$. It turns out that it will stay within $3 \%$ of $I_{\text {th }}$ as long as the workfunction variation is under 6.7 times the thermal temperature.

We use this feature of the OML blurring in our determination of the plasma potential. In other words, we locate the plasma potential by finding the potential where the collected current is equal to the electron thermal current (multiplied by the area of the probe). The cross-hair in Fig. 10 illustrates that procedure. As for the electron temperature, we may safely determine it based on the inverse of the slope of the retardation region within the linear part of Fig.11(b), that is, beyond the reach of the blurring effect of the workfunction variation, as the rightmost linear fit shows on Fig. 10. The leftmost linear fit shown on the same figure is identified with a population of warmer electrons, which account for just a few percent of the total electron population. 


\section{Experimental Results \& Analysis}

Our results are presented here in four parts: the reference cylinder, the solid tapes, the slotted tapes, and finally, a comparison of the solid and slotted tapes. All results are presented in normalized form. The collected current is normalized to the electron thermal current collected at the plasma potential, which is given by

$$
A_{p} I_{\mathrm{the}}=A_{p} n_{e} e \sqrt{\frac{e T_{e}}{2 \pi m_{e}}}
$$

All probes should collect this amount of current when biased to the plasma potential. The normalization of potential consists of a translation corresponding to the plasma potential, followed by a scaling by the inverse of the electron temperature, $T_{e}$. An OML theoretical plot, scaled this way, would result in Eq.(3) and would be independent of temperature, as discussed in the introduction. This normalization provides a means to evaluate the performance of various probes by comparing them to OML theory as well as by comparing their respective "electron collection efficiency." Note that the extent of the normalized voltage axis varies from one graph to another, due to variations in the collected data as well as to the change in electron temperature which is used in the normalization of this axis.

\section{Reference Cylinder}

Fig. 12 and 13 both show the normalized results for the reference cylinder at the three distances from the plasma source. The reference cylinder at $75 \mathrm{~cm}$ is seen to collect much more current than that predicted by OML theory, by as much as $40 \%$ at a bias of $100 T_{e}$. This enhancement is seen to decrease as we move away from the thruster to $160 \mathrm{~cm}$ and $300 \mathrm{~cm}$. In fact, there is no enhancement at 300 $\mathrm{cm}$. Since the fraction of beam ions was also determined to fall off with distance $(95 \%$ at $75 \mathrm{~cm}$, $53 \%$ at $160 \mathrm{~cm}$, and $32 \%$ at $300 \mathrm{~cm}$ ), this observed enhancement could be linked to the effects of the high-speed flow. This change in enhancement level is most likely not due to the change in the effective size of the reference cylinder $(2.0,1.1$, and 0.7 Debye lengths) since decreasing the size would theoretically have the opposite effect, that is, to increase the collection efficiency rather than to decrease it as observed here.

\section{Solid Tapes}

Fig. 12 presents results for all solid tape samples and the reference cylinder at all three distances from the plasma source $(75 \mathrm{~cm}, 160 \mathrm{~cm}$, and $300 \mathrm{~cm})$. The effective tape widths, shown in terms of the Debye

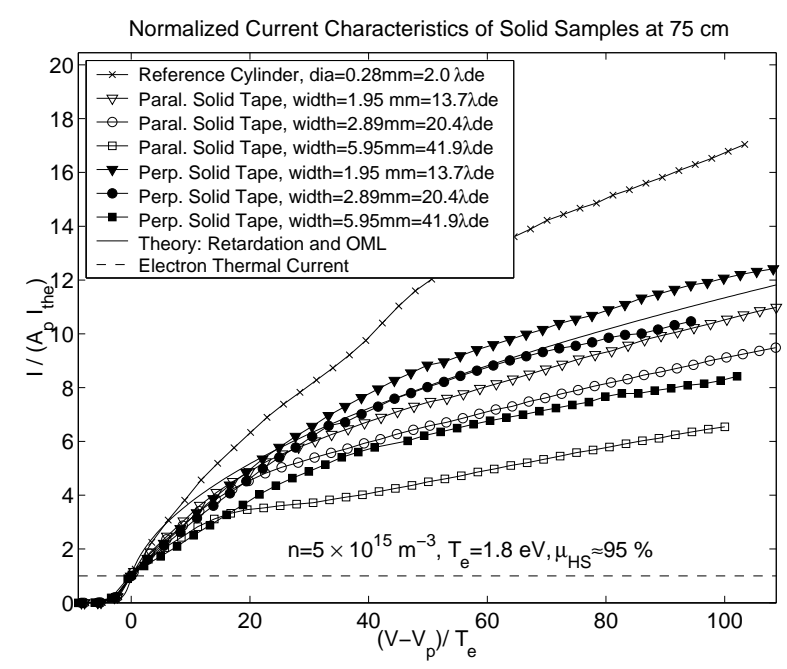

(a)

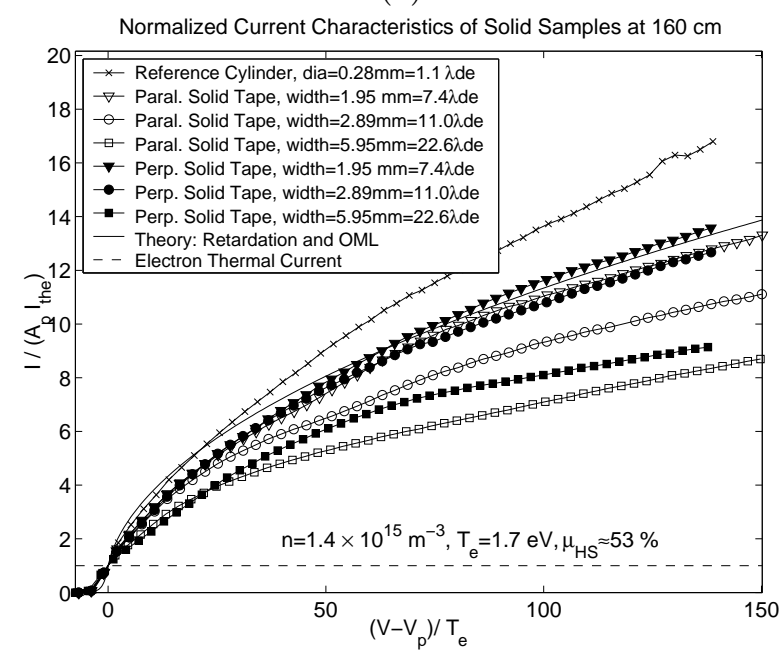

(b)

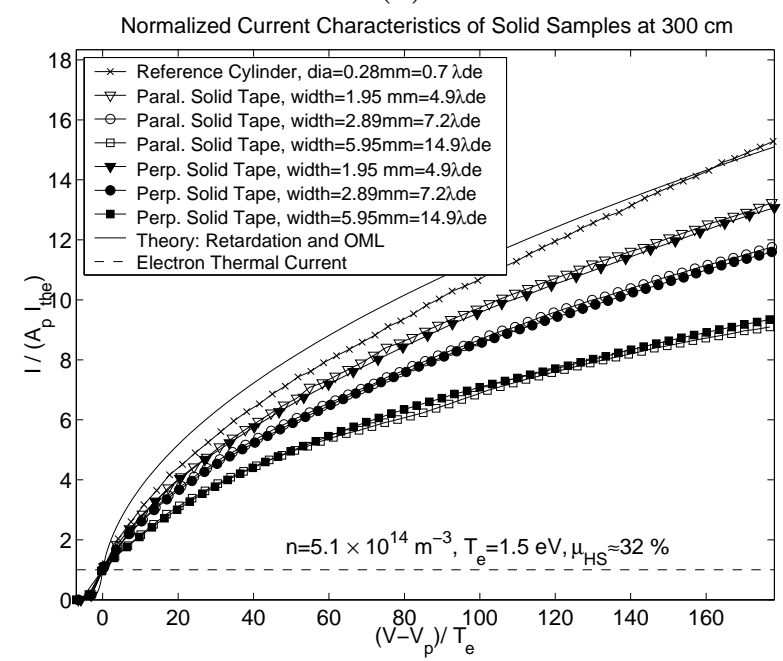

(c)

Fig. 12 Normalized I-V characteristics of parallel and perpendicular solid tapes at (a) $75 \mathrm{~cm}$, (b) $160 \mathrm{~cm}$ and (c) $300 \mathrm{~cm}$. 
lengths in the legend as well as in Table 1, span from $4.9 \lambda_{D e}$ to $41.9 \lambda_{D e}$, where $\lambda_{D e}$ is the electron Debye length.

Three major observations are noted from these results:

- First, all samples collect electrons less efficiently than the reference cylinder, as might be expected because of their larger size. Likewise, all samples are seen to collect less efficiently as the effective width of the tape is increased, regardless of the orientation. This is true whether we are looking at a mostly high-speed plasma (at $75 \mathrm{~cm}$ ) or a mostly quiescent plasma (at $300 \mathrm{~cm}$ );

- At both the $75 \mathrm{~cm}$ and $160 \mathrm{~cm}$ distances, all solid tape samples collected more current when oriented perpendicular (transverse) to the flow rather than parallel. In addition, the contrast between parallel and perpendicular results is observed to get stronger as the effective width of the tape increases. However, such a clear distinction does not show in the measurements done at $300 \mathrm{~cm}$, which is likely a consequence of the low fraction of beam ions that was measured at that location, combined with the lower effective widths of the tapes (the Debye length was highest at that location) as compared to the two other locations. The near-overlapping of the perpendicular and parallel results at $300 \mathrm{~cm}$ also serves as qualitative confirmation that the measured fraction of beam ions has dropped down as compared to that measured at $75 \mathrm{~cm}$;

- The previous observation of the increase of collected current from parallel to perpendicular is seen to occur when the probes are biased above a certain threshold, which varies from about 25 to 40 volts. This level is on the order of the estimated ion beam energy (which is somewhere between $25 \mathrm{eV}$, according to the $\mathrm{LP}$ results, and $42.9 \mathrm{eV}$, according to the LIF results). At this threshold bias, the parallel results are seen to present a "knee" which is most apparent in the widest effective tape at $75 \mathrm{~cm}$. These observation are in agreement with previous results based on a similar experiment. ${ }^{5}$

\section{Slotted Tapes}

Fig. 13 presents results for all slotted tape samples and the reference cylinder at all three distances from the plasma source. The effective center-to-center line spacings, shown in terms of the Debye lengths in the legend as well as in Table 2, span from 1.4 $\lambda_{D e}$ to $13.2 \lambda_{D e}$. It should be emphasized that the overall widths of the slotted tapes, including the gap spacings, are the same as the tape widths $(1.95 \mathrm{~mm}$, $2.89 \mathrm{~mm}$, and $5.95 \mathrm{~mm}$ ). The following are some observations regarding these results:

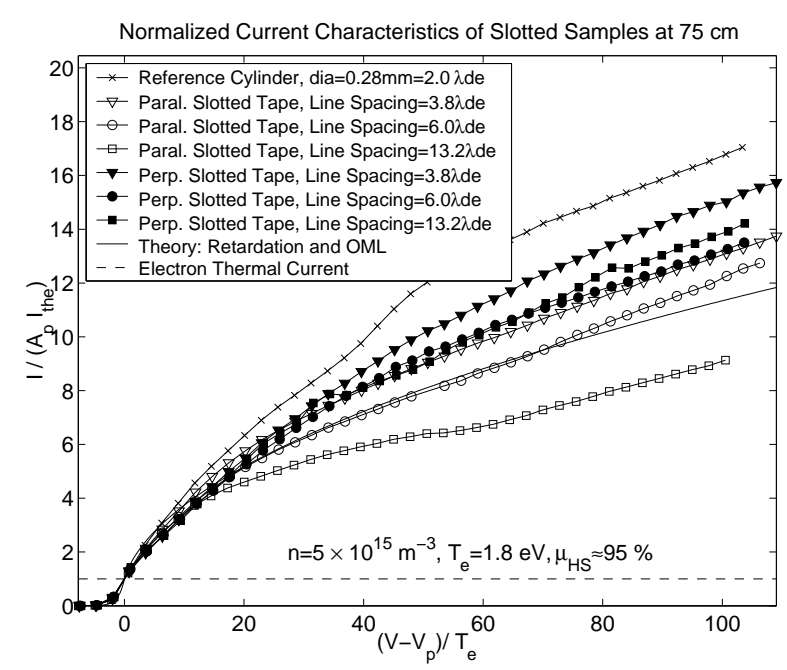

(a)

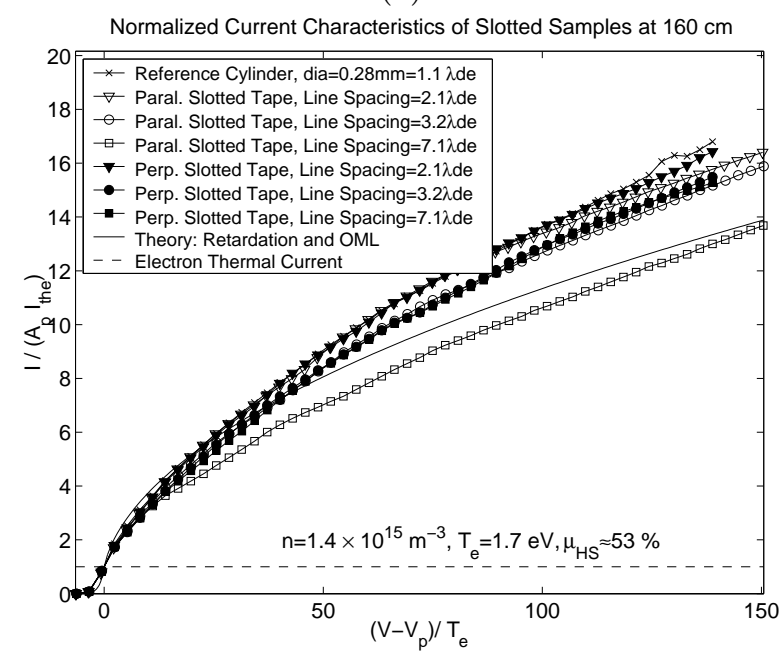

(b)

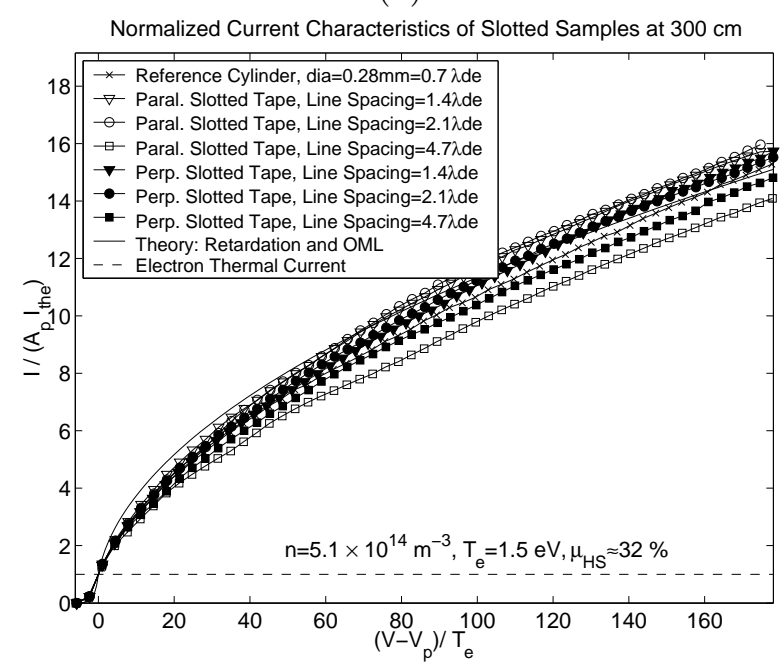

(c)

Fig. 13 Normalized I-V characteristics of parallel and perpendicular slotted tapes at (a) $75 \mathrm{~cm}$, (b) $160 \mathrm{~cm}$ and (c) $300 \mathrm{~cm}$. 
- Similar to the tape results, the slotted samples collected electrons less efficiently than the reference cylinder at $75 \mathrm{~cm}$ and $160 \mathrm{~cm}$, although they were more efficient than the solid tapes. This observation does not seem to apply for all samples at 300 $\mathrm{cm}$, where the smallest slotted tapes where actually more effective than the reference cylinder by a small margin, which might not be significant;

- The contrast between the results in the parallel and perpendicular orientations presents a similar character as that observed for the solid tapes: the perpendicular slotted tapes collect more current than their parallel counterparts. However, the reduced contrast among the results of the three perpendicular slotted tapes widths (especially at $75 \mathrm{~cm}$ and $160 \mathrm{~cm}$ ) as compared to the parallel ones is a distinct characteristic not shown by the solid tapes. Specifically, the efficiency of the widest (and most porous) slotted tape, with a line spacing of 13.2 Debye lengths at $75 \mathrm{~cm}$, is almost on par with that of the medium slotted tape $\left(6.0 \lambda_{D e}\right.$ at $\left.75 \mathrm{~cm}\right)$, and even slightly goes above it for bias potentials above $70 T_{e}$. This may indicate that the widest line spacing was close to a critical value, corresponding to the minimum efficiency, and beyond which the collection efficiency will start increasing again, up to the very wide spacing limit where all four lines become independent and collect as efficiently as the reference cylinder. We seem to have attained this limit only in the perpendicular orientation, which can be explained by the fact that the sheath elongations in the direction of the flow ${ }^{12,13}$ would cause the sheath interactions to grow stronger when aligned with (i.e., parallel to) the flow, thereby moving the critical spacing to a higher value for the parallel case. Clearly, testing of wider line spacings will be required to determine those critical values and relate them to the flow energy;

- A "knee" is most apparent in the results for the widest parallel slotted tapes at $75 \mathrm{~cm}$ and 160 $\mathrm{cm}$, around 29 and 21 volts, respectively (note that the position of the knee on the graphs need to be scaled with their respective electron temperatures), which is close to the estimated ion beam energy. The parallel and perpendicular results separate at the potential bias corresponding to the observation of this knee.

\section{Comparison of the Solid and Slotted Tapes}

Fig. 14-16 shows the same sets of results shown earlier, but with the solid and slotted tapes plotted on common graphs to ease their comparison. The absolute amount of current collected by the solid tape samples was higher than that collected by the

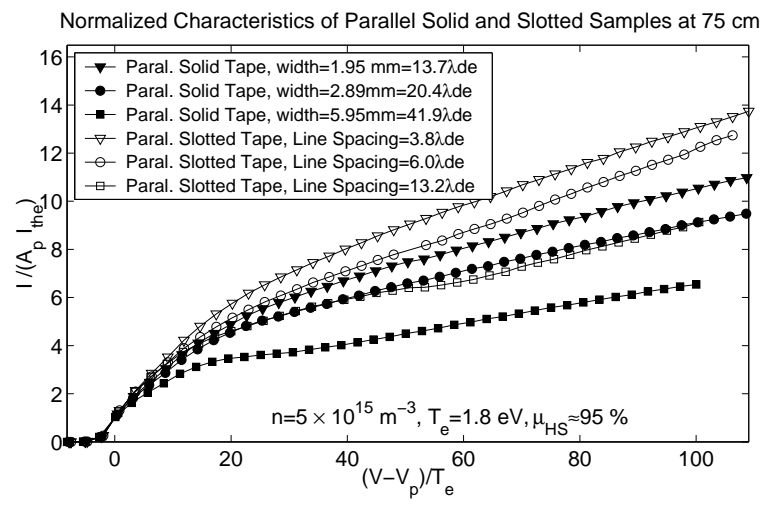

Normalized Characteristics of Perpendicular Solid and Slotted Samples at $75 \mathrm{~cm}$

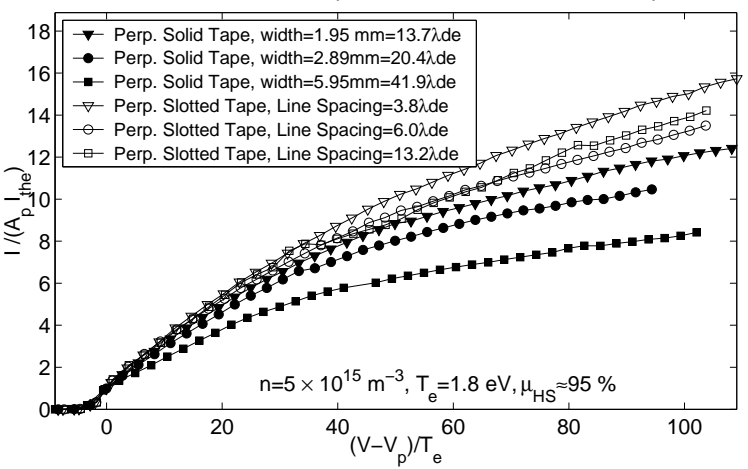

Fig. 14 Comparison of the I-V characteristics of Solid and Slotted Tapes at $75 \mathrm{~cm}$.

slotted tapes samples in all cases, as expected, and is not shown here. The slotted samples were somewhat more efficient on a per-area basis than their solid counterparts. This is true at all positions, and does not seem to be a function of the fraction of beam ions present. Hence, the slotted samples are always more efficient on a per-area basis, whether in a nonflowing or flowing plasma. For example, at $75 \mathrm{~cm}$, the parallel wide slotted tape collected about $37 \%$ of the current collected by the parallel wide solid tape at a normalized bias of $80 T_{e}$, a higher fraction than the porosity of the sample, which was $28 \%$.

\section{Present Status and Conclusions}

Several conclusions can be drawn from the analysis of the results:

1. The plasma flow leads to current enhancements over that predicted by the orbital-motionlimited theory;

2. the electron collection efficiency of solid tapes (on a per area basis) decreases as the width of the tape is increased;

3. beyond a threshold bias close to the beam energy, solid and slotted tapes both collect more current when oriented transverse to the flow; 


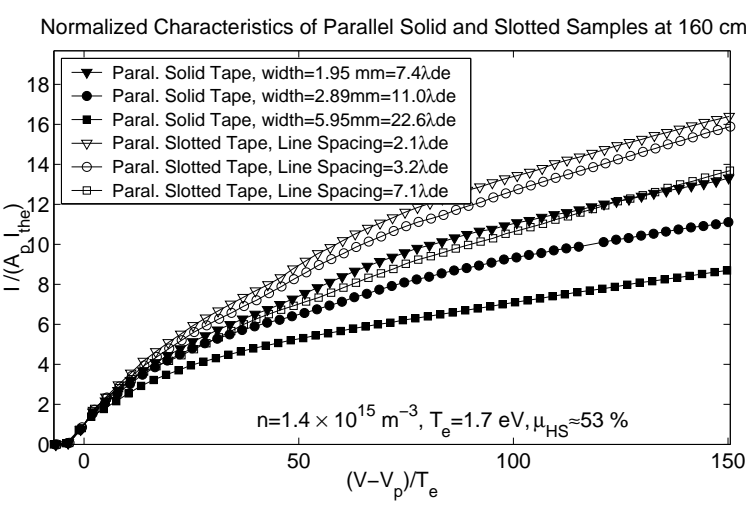

Normalized Characteristics of Perpendicular Solid and Slotted Samples at $160 \mathrm{~cm}$

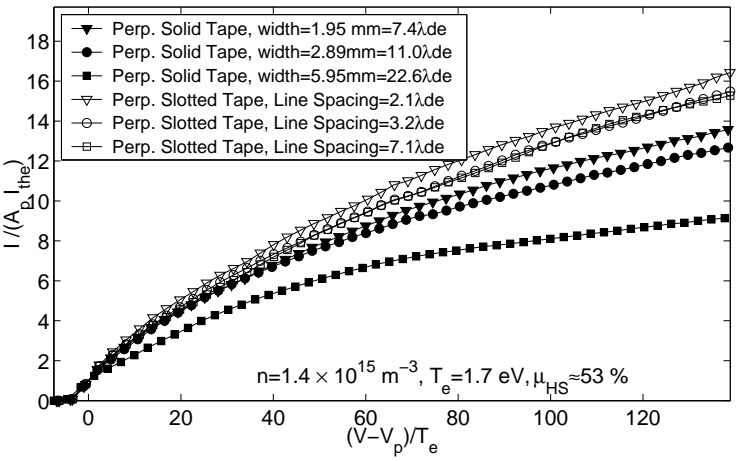

Fig. 15 Comparison of the I-V characteristics of Solid and Slotted Tapes at $160 \mathrm{~cm}$.

4. equivalent width slotted tapes are more efficient electron collectors than solid tapes on a per area basis;

5. our data suggests the electron collection efficiency of slotted tapes decreases with increasing line spacing until a possible minimum efficiency is attained, beyond which it is expected to start increasing again. The minimum was attained in the case of the samples oriented transverse to the flow, but not in the case of the samples aligned with the flow, for which the critical spacing is likely higher due to an increased sheath interaction radius of each line caused by the elongation of the sheath associated with plasma flow.

Further experiments are needed to accurately quantify the observed effects. In addition, larger line spacings should be tested in both the parallel and perpendicular orientations to verify the existence of and quantify the critical spacing that correspond to a minimum collection efficiency. Finally, a lower background pressure might help improving the survival of the ion beam out to 3 meters, and could possibly be achieved with the use of additional cryopumps.
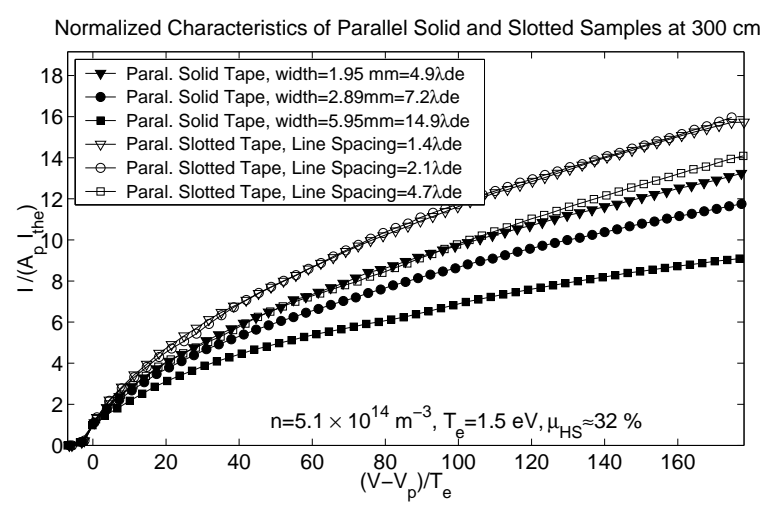

Normalized Characteristics of Perpendicular Solid and Slotted Samples at $300 \mathrm{~cm}$

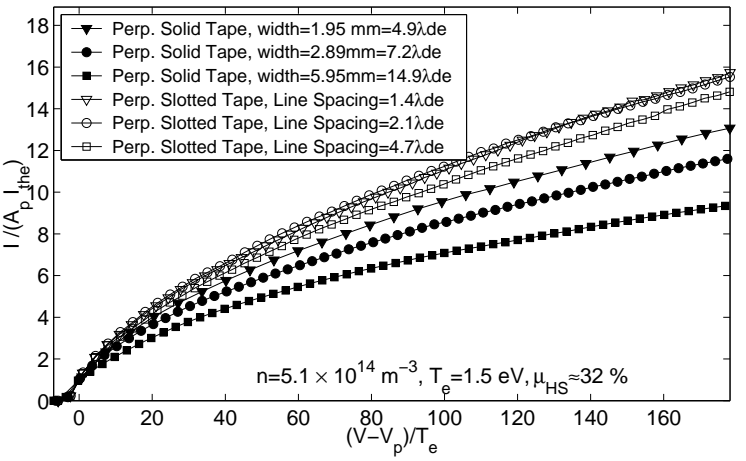

Fig. 16 Comparison of the I-V characteristics of Solid and Slotted Tapes at $300 \mathrm{~cm}$.

\section{Acknowledgments}

The authors would like to thank Prof. A.D. Gallimore, T. Smith, D. Herman, and the PEPL research group for support in performing those tests in PEPL's Large Vacuum Test Facility. É.C. acknowledges the scholarship support of the Horace H. Rackham School of Graduate Studies at the University of Michigan, the Natural Sciences and Engineering Research Council of Canada and the Communications Research Centre(Canada).

\section{References}

${ }^{1}$ Sanmartín, J., Martínez-Sánchez, M., and Ahedo, E., "Bare Wire Anodes for Electrodynamic Tethers," Journal of Propulsion and Power, Vol. 9, No. 3, 1993, pp. 353-360.

${ }^{2}$ Johnson, L., Gilchrist, B. E., Estes, R. D., Lorenzini, E., and Ballance, J., "Propulsive Small Expendable Deployer System (ProSEDS) Space Experiment," AIAA Paper AIAA98-4035, 1998.

${ }^{3}$ Noord, J. V. and Strumfels, R., "Electrodynamic Tether Optimization for the STEP-AirSEDS Mission," AIAA Paper AIAA-2001-3980, 2001.

${ }^{4}$ Mott-Smith, H. and Langmuir, I., "The Theory of Collectors in Gaseous Discharges," Physical Review, Vol. 28, 1926 , pp. $727-763$.

${ }^{5}$ Gilchrist, B. E., Bilén, S. G., Choinière, E., Gallimore, A. D., and Smith, T. B., "Analysis of Chamber Simulations of Long Collecting Probes in High-Speed Dense Plasmas," 
IEEE Transactions on Plasma Science, Vol. 30, No. 5, October 2002, pp. 2023-2034.

${ }^{6}$ Haas, J. M., Gulczinski, F. S. II, Gallimore, A. D., Spanjers, G. G., and Spores, R. A., "Performance characteristics of a $5 \mathrm{~kW}$ laboratory Hall thruster," AIAA Paper AIAA-98$3503,1998$.

${ }^{7}$ Gilchrist, B. E., Bilén, S., Choiniere, E., and Gallimore, A. D., "Laboratory Experiments of Current Collection to Long Tape Probes Using a High-Speed Plasma Relevant to Bare Electrodynamic Tethers: Calibration and Initial Solid and Slotted Tape Measurements," Tech. Rep., University of Michigan, 2002.

${ }^{8}$ Williams, G. J. Jr., Smith, T. B., Domonkos, M. T., Shand, K. J., Gallimore, A. D., and Drake, R. P., "Laser Induced Fluorescence Measurement of Ion Velocities in the Plume of a Hall Effect Thruster," AIAA Paper AIAA-992862, 1999.

${ }^{9}$ Smith, T. B., Herman, D. A., Gallimore, A. D., and Drake, R. P., "Deconvolution of Axial Velocity Distributions from Hall Thruster LIF Spectra," Paper IEPC-01-0019 presented at the 27th International Electric Propulsion Conference, 1999.

${ }^{10}$ Chung, P. M., Talbot, L., and Touryan, K. J., Electric Probes in Stationary and Flowing Plasmas: Theory and Application, Springler-Verlag, 1975.

${ }^{11}$ Hoegy, W., and Wharton, L., "Current to a Moving Cylindrical Electrostatic Probe," Journal of Applied Physics, Vol. 44, No. 12, December 1973, pp. 5365-5371.

${ }^{12}$ Choinière, E., and Gilchrist, B. E., "Electron Collection to Arbitrarily Shaped Electrodynamic Tethers in Flowing Plasmas: a Kinetic Model - Validation for Circular Crosssections," AIAA Paper AIAA-2002-4050, 2002.

${ }^{13}$ Choinière, E., and Gilchrist, B. E., "Modeling Long Probes in Flowing Plasmas using KiPS-2D, a Novel SteadyState Vlasov Solver," AIAA Paper AIAA-2003-5098, 2003.

${ }^{14}$ Brace, L. H., Measurement Techniques in Space Plasmas: Particles, Langmuir Probe Measurements in the Ionosphere, American Geophysical Union, 1998, pp. 23-35. 